\title{
Linx
}

Revue des linguistes de l'université Paris X Nanterre

$61 \mid 2009$

Entre rection et incidence : des constructions verbales atypiques?

\section{Constructionalisation et structure informationnelle. Quand la grammaticalisation ne suffit pas pour expliquer tu vois}

\section{Catherine Bolly}

\section{OpenEdition}

\section{Journals}

Édition électronique

URL : http://journals.openedition.org/linx/1342

DOI : $10.4000 /$ linx.1342

ISSN : 2118-9692

\section{Éditeur}

Presses universitaires de Paris Nanterre

Édition imprimée

Date de publication : 1 juin 2009

Pagination : 103-130

ISSN : 0246-8743

\section{Référence électronique}

Catherine Bolly, «Constructionalisation et structure informationnelle. Quand la grammaticalisation ne suffit pas pour expliquer tu vois », Linx [En ligne], 61 | 2009, mis en ligne le 01 juin 2013, consulté le 19 avril 2019. URL : http://journals.openedition.org/linx/1342 ; DOI : 10.4000/linx.1342 


\title{
Constructionalisation et structure informationnelle. Quand la grammaticalisation ne suffit pas pour expliquer $t u$ vois
}

\author{
Catherine Bolly \\ F.R.S.-FNRS \& Université catholique de Louvain
}

\section{Introduction}

Dans cet article, nous proposons d'aborder le processus évolutif de la construction parenthétique tu vois en prenant appui à la fois sur le champ d'étude de la grammaticalisation et sur l'étude de l'évolution de la structure informationnelle (du français préclassique jusqu'à nos jours). Pour mettre en perspective cette double approche, nous adoptons une méthode d'analyse paramétrique et statistique de données de corpus. Les principaux paramètres pris en compte sont des critères linguistiques sémantico-pragmatiques (déplacement sémantique, implication de l'interlocuteur dans le discours) et formels (position syntaxique de la construction, présence ou non de marqueurs de discours à l'initiale, nature syntaxique de la portée). Avant d'aborder la partie d'analyse des données, nous proposons tout d'abord une définition des marqueurs de discours et des constructions parenthétiques, en accordant une attention particulière au rôle joué par le contexte dans le processus évolutif de ces constructions. 


\section{Les constructions parenthétiques : des marqueurs de discours complexes}

\subsection{Marqueurs de discours}

Comme différents auteurs le soulignent (Hansen, 1998, pp. 65-66 ; Schourup, 1999, p. 228 ; Lewis, 2006, pp. 43-44), la question de savoir si les marqueurs de discours constituent en eux-mêmes une catégorie linguistique est encore actuellement sujette à controverse. Cette difficulté tient principalement au fait que les marqueurs de discours ne se définissent pas à partir de critères strictement (morpho-)syntaxiques, mais avant tout à partir de critères pragmatiques et fonctionnels : « discourse marker refers to a range of form-function mappings, rather than to a closed set of forms » (Lewis, 2006, p. 44). Les marqueurs de discours sont en effet plurifonctionnels et multi-catégoriels : (i) une même unité (par ex. alors, bon, tiens) peut avoir une fonction micro-syntaxique quand elle agit sur le contenu propositionnel de l'énoncé, ou une fonction macrosyntaxique (discourse-marking use - cf. Hansen 1998 et Lewis 2006) quand elle agit au niveau de l'énonciation (au sens de Benveniste 1966) ; (ii) les marqueurs de discours, entendus dans leur acception large de marqueurs pragmatiques ( $c$. Brinton 1996), relèvent de catégories syntaxiques diverses : interjections et particules (par ex. euh, ben, $\mathrm{mmm}$ ), adverbes (par ex. franchement, enfin), syntagmes verbaux (par ex. vous savez, prenons l'exemple de), syntagmes nominaux (par ex. style, genre), conjonctions de subordination (par ex. parce que) et de coordination (par ex. et, donc, mais). Au-delà de cette hétérogénéité, on s'accorde néanmoins ( $c f$. Hansen 1998) sur le fait que les marqueurs de discours sont des indices de cohérence discursive dont la portée est variable: "The speaker makes use of them to segment, recover, organize, and reformulate the information provided to the hearer, but also to share common ground, assumptions and presuppositions with him/her» (González, 2008, p. 2). On reconnait en outre aux marqueurs de discours certaines propriétés qui, si elles ne sont ni nécessaires ni suffisantes pour les caractériser de manière homogène, s'avèrent pertinentes quand on les aborde de manière non isolée. Selon Hansen (1998, p. 62), les marqueurs de discours sont ainsi reconnus comme tels quand ils combinent une série de critères syntaxiques, sémantiques et/ou fonctionnels (voire informationnels - $f$. Lewis 2006). Parmi ces critères, nous en énonçons ici les principaux qui font l'objet d'un relatif consensus dans le domaine. D’un point de vue général, les marqueurs discursifs sont le plus souvent des indices du caractère informel des textes et sont fréquents dans l'usage (surtout à l'oral, mais aussi à l'écrit). Dans certains contextes, par exemple dans des situations qui génèrent du stress ou un sentiment d'insécurité, les marqueurs de discours peuvent être des traces verbales du comportement (hyper)modalisateur du locuteur (cf. Schneider 2007 ; Nespoulous, 2010, pp. 92-93) cf. (1).

(1) et tu vois en même temps c'est je me dis comme ce ser/ ce serait encore mieux si euh / s'il n'y avait rien eu quoi tu vois / en même temps voilà il y a on a des des / des / enfin la situation faisait que tu vois on s'est connus euh super jeunes aussi quelque part tu vois eub / début rhéto enfin en début rbéto tu es encore un peu ado quoi tu vois [...] (Oral, Contemporain, Valibel)

Les marqueurs de discours dépendent donc fortement du contexte communicationnel dans lequel ils apparaissent. D’un point de vue linguistique, ce sont généralement des 
formes courtes qui sont prosodiquement autonomes et qui peuvent présenter une réduction phonologique (par ex. ben, 'fin, t'sais). Les marqueurs de discours se trouvent le plus souvent en position extra-propositionnelle, i.e. en périphérie de la syntaxe (cf. infra), avec une préférence pour la position à l'initiale, bien qu'ils puissent aussi se trouver en position médiane (par ex. «donc» et «tu sais» dans (2)) ou en finale d'énoncé (par ex. « quoi » et « hein » dans (2)).

(2) je m'occupe de / de revalidations lourdes et y compris de / de tout ce qui est réinsertion socioprofessionnelle / des traumatisés crâniens donc avec / altérations cognitives troubles tu sais eub mnésiques attentionnels enfin brindezingues quoi bein (Oral, Contemporain, Valibel)

Leur intégration micro-syntaxique est faible, voire nulle, ce qui va de pair avec leur optionalité grammaticale et leur tendance à être mobiles au sein de l'énoncé qui les héberge : l'absence de marqueur de discours ne rend pas un énoncé agrammatical ou inintelligible. S'ils sont optionnels sur le plan grammatical, ils sont cependant nécessaires pragmatiquement (pragmatically required - cf. Brinton, 2008, p. 14 ; communicative obligatoriness cf. Diewald 2010). Sémantiquement, leur contenu est procédural plutôt que conceptuel (perspective cognitive) : les marqueurs de discours sont des unités indexicales, qui guident l'interlocuteur/scripteur sur la façon dont il faut organiser et manipuler l'information conceptuelle. Leur sémantisme peut être vu comme le résultat d'un processus de déplacement par aréférenciation ou abstraction (blanchiment sémantique ou désémantisation) (perspective diachronique). Si l'on en croit Brinton (2008), les constructions parenthétiques (appelées comment clauses par l'auteur) partagent la plupart des caractéristiques attribuées aux marqueurs de discours (appelés pragmatic markers par Brinton 1996). Comme nous le montrons plus loin, les constructions parenthétiques ont cependant ceci de particulier qu'elles ont une structure propositionnelle (i.e. clausale) dont l'emploi s'est conventionnalisé dans l'usage. En tant qu'unités collocationnelles (Bolly à par.), elles possèdent un certain nombre de propriétés qui les distinguent des marqueurs de discours monolexicaux, notamment une forte collocabilité (tendance à la contiguïté, à la coalescence et à la fusion de leurs constituants) qui n'exclut toutefois pas une certaine flexibilité syntagmatique (possibilité d'insertion entre les constituants de la construction, possibilité de substitution quasi-synonymique des paradigmes) (cf. la notion de persistence of grammatical conditioning - Torres Cacoullos \& Walker 2009). C'est cette spécificité par rapport aux marqueurs de discours monolexicaux qui les rend difficilement catégorisables de manière univoque (Bolly à par.) soit dans le domaine de la (micro-)syntaxe, soit dans celui de la macro-syntaxe (Blanche-Benveniste 2003 ; Berrendonner 2002, 2004).

\subsection{Constructions parenthétiques}

Reflétant la diversité des approches sur la question et le foisonnement terminologique qui en résulte ${ }^{1}$, les constructions parenthétiques ont été désignées en français par les termes d'incises (Cornulier 1978; Blanche-Benveniste 1989), d'incidentes (Marandin 1998), de propositions parenthétiques ou marqueurs de discours propositionnels (Andersen 1997, 2007) ou encore de clauses parenthétiques (réduites) (Schneider 2007, ici-même). Ces termes,

\footnotetext{
${ }^{1}$ Pour un état plus complet de la question, nous renvoyons à l'ouvrage de Schneider (2007).
} 
s'ils ne sont pas tout à fait équivalents, prennent néanmoins tous pour objet d'étude les syntagmes verbaux (par ex. je crois) qui semblent fonctionner dans certains contextes comme des indicateurs de subjectivité (Benveniste 1966), autrement dit comme des modalisateurs permettant au locuteur de produire un énoncé en même temps qu'il exprime son attitude ou son opinion par rapport au contenu de cet énoncé.

En tant que modalisateur de telle ou telle proposition complétive ou qualifiée en incise, je crois, je pense, je dirais manifestent symptomatiquement que le locuteur est relativement assuré, mais pas certain de ce qui va suivre, soi-disant qu'il n'y croit pas, c'est-à-dire qu'il s'agit d'une reformulation, disons d'une approximation ou correction. Ce genre de dérivation formulaire s'appuie sur la parenthétisation progressive d'un verbe de parole ou d'attitude propositionnelle (au sens de Urmson 1952 et de Reinhart 1983), associé à un affaiblissement de sa force descriptive. Il en va de même des marques d'adresses comme tu sais, tu penses, tu vois, ainsi que de bien d'autres formulations dont nous n'allons bien entendu pas faire ici le tour. (Perrin, 2008, p. 173)

Dans la lignée des chercheurs qui étudient la syntaxe de l'oral, ces constructions sont perçues avant tout comme des structures syntaxiques construites avec un verbe dont la fonction régissante est plus faible que celle des verbes pleins à forte rection (Blanche-Benveniste 1989 ; Blanche-Benveniste \& Willems 2007 ; Willems \& Blanche-Benveniste 2010). Dans une perspective plus fonctionnaliste de l'analyse du discours, ces constructions sont décrites avant tout à partir de la fonction sémanticopragmatique qu'elles remplissent en discours. C'est ainsi que Dehé \& Wichmann (2010a, 2010b), qui enrichissent l'analyse par le recours à des critères prosodiques, mettent au jour trois fonctions pour les constructions parenthétiques (à la $1^{\mathrm{e}} \mathrm{p}$. du singulier en anglais parlé): (i) une fonction référentielle, quand la construction conserve son statut de proposition principale avec ou sans particule complétive (main clause); (ii) une fonction de commentaire (comment clauses), allant de pair avec un glissement sémantique par abstraction et l'acquisition d'une fonction proprement interactionnelle, interpersonnelle ou textuelle ; (iii) une fonction de ponctuant discursif (verbal filler), dans le cas des emplois très figés (formulaic). À noter que cette tripartition est envisagée de manière graduelle en synchronie par les auteurs. Schneider (2007) reconnaît quant à lui trois fonctions principales aux clauses parenthétiques (réduites et non réduites) : (i) une fonction phatique (par ex. vous savez); (ii) une fonction liée au discours rapporté (par ex. dit-il); (iii) une fonction modalisatrice (par ex. disons, je veux dire, je sais pas, je me rappelle). Parmi les clauses à fonction modalisatrice, l'auteur distingue quatre fonctions principales pour les constructions parenthétiques réduites (en français, espagnol et italien) : (i) une fonction référentielle, qui concerne les clauses qui portent sur le contenu propositionnel tout en le précisant (par ex. disons); (ii) une fonction performative, qui concerne les clauses qui indiquent de manière directe ou indirecte la force illocutoire, tout en portant en même temps sur le contenu propositionnel et/ou sur l'implication du locuteur (par ex. je veux dire, je dirais) ; (iii) une fonction d'atténuation directe de l'implication du locuteur, qui concerne principalement les clauses avec verbes de croyance (par ex. je sais pas, je suppose, je pense); (iv) une fonction d'atténuation indirecte de l'implication du locuteur par le renvoi à la source de l'information (par ex. je me rappelle, il me semble). 
Pour la présente étude, nous prendrons appui sur la définition que Schneider a donnée des clauses parenthétiques réduites (2007, p. 1), en y incluant les constructions à l'initiale d'énoncé (à l'instar de Urmson 1952). Cette définition large du phénomène a l'avantage de ne poser aucun a priori quant au rôle joué par la position sur la fonction discursive des constructions étudiées. Sur le plan formel, les constructions parenthétiques possèdent plusieurs propriétés qui, comme nous l'avons évoqué précédemment, se rapprochent des propriétés des marqueurs de discours. Bien que constituées d'un verbe fini, elles ne correspondent ni à une clause principale ni à une clause subordonnée, mais se trouvent en périphérie de la syntaxe (cf. infra) à la manière des adverbiaux de phrase. Partant, elles sont grammaticalement optionnelles et leur position est relativement libre : elles peuvent être insérées, antéposées ou postposées par rapport à leur " hôte » (i.e. par rapport à l'énoncé, à la clause ou au syntagme qui les héberge). En outre, les constructions parenthétiques sont asyndétiques, autrement dit, elles ne sont pas connectées explicitement à leur hôte par un segmenteur (par ex. que, si, combien) et ne sont reliées formellement à celui-ci que par une relation de contigüité (voir aussi Kaltenböck, 2007, p. 3). Les constructions parenthétiques (contrairement aux incises) sont réduites, c'est-à-dire qu'il leur manque un argument valenciel pour pouvoir être considérées comme des clauses complètes. Sur le plan sémantico-pragmatique, on considère généralement que l'argument absent est récupérable dans le contexte immédiat, à savoir dans l'unité « hôte » (voir aussi Kaltenböck, 2007, p. 4) : les constructions parenthétiques réduites seraient donc reliées à leur hôte par une relation qui n'est pas d'ordre (morpho-)syntaxique, mais bien sémantico-pragmatique. Notons que nous utilisons le terme de construction parenthétique de manière générique pour désigner ce type d'unités linguistiques dans la présente étude.

Ces considérations nous poussent à penser (Bolly à par.) que les constructions parenthétiques sont bien des marqueurs de discours complexes : «A pragmatic marker is defined as a phonologically short item that is not syntactically connected to the rest of the clause (i.e., is parenthetical), and has little or no referential meaning but serves pragmatic or procedural purposes » (Brinton, 2008, p. 1).

L'objet d'étude ayant été défini, nous nous attacherons dans le point qui suit à le replacer dans le cadre théorique qui a servi de point d'ancrage à l'analyse diachronique sur corpus, à savoir le champ d'étude de la grammaticalisation et, de manière plus large, celui du changement linguistique. Une attention particulière sera portée au rôle joué par le contexte, d'une part, dans les processus de grammaticalisation et, d'autre part, dans l'évolution de la structure informationnelle.

\section{Evolution des constructions parenthétiques}

Nous avons choisi de nous centrer dans cet article sur le rôle joué par le contexte dans le processus évolutif des constructions parenthétiques pour différentes raisons. Tout d'abord, comme l'ont souligné Langacker (1987) et Bybee (2003), on s'accorde actuellement sur le fait que la fréquence des mots dans l'usage, et en particulier la répétition de ceux-ci dans des contextes spécifiques, joue un rôle prépondérant dans l'évolution sémantico-pragmatique et la conventionnalisation de ces emplois : «With repeated use, a novel structure becomes progressively entrenched, 
to the point of becoming a unit ; moreover, units are variably entrenched depending on the frequency of their occurrence » (Langacker, 1987, p. 59). En outre, les parenthétiques sont par définition des unités phraséologiques (Torres Cacoullos \& Walker 2009, 2011 ; Bolly à par.) ou des constructions au sens de la grammaire constructionnelle (Goldberg 1995 ; François \& Legallois 2006) qui montrent un certain degré de collocabilité entre leurs constituants, ce qui implique la nécessité de les étudier de manière non isolée et de prendre en considération leur environnement linguistique immédiat. Enfin, on reconnaît traditionnellement l'importance de l'influence de l'ordre des mots et de la position des unités linguistiques au sein des énoncés sur leur fonction linguistique, syntaxique ou discursive (Auer 1996 ; Aijmer 2007), mais aussi sur leur fonction dans l’organisation informationnelle du discours (Charolles 2003 ; Prévost 2003a).

\subsection{Contexte, grammaticalisation et pragmaticalisation}

En tant que processus relevant du changement linguistique, la grammaticalisation désigne un mouvement diachronique qui voit certaines unités migrer de la sphère lexicale vers la sphère grammaticale (par ex. le nom latin homo « être humain » qui a donné lieu au pronom impersonnel on), de la sphère grammaticale vers un emploi davantage grammatical (par ex. le verbe de mouvement aller qui a donné lieu au futur périphrastique), voire vers la sphère pragmatique (par ex. écoute donc qui a donné lieu à coudon en français québécois - cf. Dostie 2004). En tant que marqueurs de discours, on peut considérer que les parenthétiques seraient le résultat d'un processus de pragmaticalisation (Erman \& Kotsinas 1993 ; Dostie 2004) qui voit certaines constructions verbales migrer de la sphère lexico-grammaticale, où elles remplissent une fonction micro-syntaxique au niveau des contenus propositionnels, vers la sphère pragmatique, où leur fonction macro-syntaxique est davantage liée à la situation effective de communication langagière dans laquelle elles sont produites. Plusieurs principes (Hopper 1991) et paramètres (Lehmann 1995) ont été mis au jour pour caractériser le processus de grammaticalisation. Reformulés plus récemment par Marchello-Nizia (2006), nous les résumons ci-dessous :

- le caractère obligatoire, la progressivité et l'unidirectionnalité seraient des caractéristiques spécifiques aux grammaticalisations (à noter que Prévost (2003b) préfère parler d'irréversibilité) ;

- la grammaticalisation s'accompagnerait d'un déplacement sémantique par abstraction, allant souvent de pair avec un processus d'(inter)subjectification (Traugott 1982, 2010) et/ou de métaphorisation-métonymisation ;

- d'un point de vue formel, les unités en cours de grammaticalisation peuvent subir un affaiblissement phonologique, prosodique et accentuel ;

- syntaxiquement, elles se caractérisent (i) par une décatégorisation morphologique (avec recatégorisation éventuelle), (ii) par un figement syntaxique qui aboutit le plus souvent à une soudure, ou du moins à une coalescence ou une contigüité ( $c f$. la notion de paradigmatisation - Marchello-Nizia, 2006, p. 41), (iii) par un mécanisme de réanalyse, qui consiste en un «changement syntaxique dans la structure d'une expression ou d'une classe d'expressions, un 'reparenthésage' de ses éléments en quelque sorte, sans que cela se manifeste dans sa structure de surface » (Marchello- 
Nizia, 2006, p. 43), donnant lieu à des interprétations nouvelles de la structure qui apparait dans de nouveaux contextes.

Nous ne reviendrons pas ici sur le bienfondé de ces principes qui, bien que critiquables à plusieurs égards (Béguelin 2009, 2010) et notamment du point de vue du principe de l'unidirectionnalité (e.a. Traugott 2001 ; Prévost 2003b ; Haspelmath 2004 ; Norde 2009), ont l'avantage de fournir des outils opérationnels et pertinents pour décrire l'évolution de certaines unités linguistiques.

Par ailleurs, on reconnaît que le contexte joue un rôle crucial sur le processus évolutif des unités qui sont en cours de grammaticalisation/pragmaticalisation (cf. Heine 2002 ; Diewald 2006). C'est ainsi que selon Heine (2002), le processus de grammaticalisation se ferait en quatre phases qui peuvent coexister durant une même période. Au stade initial, le mot susceptible de se grammaticaliser est employé dans son sens originel dans tous ses emplois. Au second stade, qui correspond au contexte de transition ("bridging context»), le mot apparaittrait dans un nouveau contexte qui permettrait à son tour l'émergence d'un nouveau sens. Ce second stade se caractérise par la coexistence de plusieurs réalisations sémantiques concurrentes d'une même unité dans un contexte nouveau, dès lors ambigu. Autrement dit, les contextes ambigus favorisent l'apparition de sens nouveaux : «C'est donc d'abord par la modification des contextes dans lesquels le mot se rencontre que le changement apparait » (MarchelloNizia, 2006, p. 23). Au troisième stade, appelé contexte de passage (" switch context»), la nouvelle signification apparait comme étant incompatible avec le sens initial dans certains contextes (relégué à l'arrière-plan, mais toujours accessible). Et c'est le nouveau sens qui permet à son tour de créer de nouveaux contextes, cette fois non ambigus. Au quatrième et dernier stade de conventionnalisation (" conventionalisation »), l'usage ancien est encore présent, mais «n'est plus senti comme ayant aucune parenté avec le nouveau morphème » (Marchello-Nizia, 2006, p. 22). Il y a primauté du sens nouveau sur l'ancienne signification.

Nous verrons lors de l'analyse sur corpus, en quoi le contexte tend à jouer un rôle sur l'évolution des constructions parenthétiques. Mais il s'avère essentiel de donner au préalable un aperçu des études qui ont porté sur l'évolution des parenthétiques, en s'attardant davantage sur les études qui tiennent compte de la spécificité collocationnelle (ou constructionnelle) de telles unités.

\subsection{Constructionalisation des parenthétiques}

$[\mathrm{t}$ has been shown that a new grammatical function does not arise homogeneously in all uses of the linguistic item concerned, but is bound in its origin to specific linguistic « contexts » or « constructions » (Diewald, 2006, p. 3).

La plupart des études portant sur l'évolution des parenthétiques prennent pour acquis que ces constructions sont par nature elliptiques et qu'elles proviennent, tant en anglais qu'en français, d'une proposition principale constituée d'un syntagme verbal avec verbe fléchi (matrix clause), régissant elle-même une proposition complétive introduite par que (ou that en anglais) (3) (e.a. Thompson \& Mulac 1991; BlancheBenveniste \& Willems 2007 ; Willems \& Blanche-Benveniste 2010). Lors d'une phase 
d'évolution intermédiaire, il y aurait ainsi ellipse de la particule complétive que (ou that) (Ex. 4), avant que la construction ne se grammaticalise (5).

(3) Elle m'a supplié de lui laisser le tems de se retirer. On ne devoit pas savoir qu'elle fût dans la maison. Charmante fille! Tu vois, Belford, qu'elle ne pense plus à me quitter. (Litt., Classique, L'Abbé Prévost, Lettres angloises on Histoire de miss Clarisse Harlove, 1751, Frantext)

(4) [...] et j'ai répondu pour toi, comme si je t'avois vu, [...] que d'ailleurs tu avois une petite indisposition, qui exigeoit beaucoup de circonspection dans les visites aux femmes qu'on aime. Ne va pas me démentir! Par-là, tu vois bien, je te donne un temps plus que suffisant pour que ton Obscurophile te sorte par les yeux. (Litt., Classique, Nicolas Rétif de la Bretonne, Le Paysan perverti ou les Dangers de la ville, 1776, Frantext)

(5) L1 et en cinquante-neuf mes parents construisaient une maison / ici à Paliseul / tu vois alors j'ai travaillé disons six mois / / euh / là-bas dans un petit village qui s'appelle Framont bien sûr / et puis au Nouvel-An je suis venu avec mes parents ici quoi (Oral, Contemporain, Valibel)

Il ressort par ailleurs des études de Torres Cacoullos et ses collaborateurs (Bybee \& Torres Cacoullos 2009 ; Torres Cacoullos \& Walker 2009, 2011), qui s'inscrivent dans la lignée des grammaires émergentes (Emergent Grammar - Hopper 1987 ; Bybee 2006), que les constructions parenthétiques conservent non seulement une trace du contenu sémantique des unités lexicales qui les constituent (lexical retention), mais aussi une trace du comportement syntaxique de leur structure verbale (grammatical retention).

While the literature on grammaticization has focused on semantic retention, we propose grammatical retention, in that vestiges of the linguistic conditioning of productive grammatical constructions persist even in fixed discourse formulas originating from those constructions. In other words, constraints on the source construction persist in the discourse formula. (Torres Cacoullos \& Walker, 2009, p. 2)

En d'autres termes, cette approche conçoit les parenthétiques comme étant le produit d'un processus graduel de constructionalisation (voir aussi Traugott 2008 ; Lewis 2011 ; Van Bogaert 2011). Autrement dit encore, les parenthétiques seraient des unités phraséologiques semi-figées qui conserveraient une trace de leur compositionnalité sémantique et un certain degré de flexibilité syntaxique (Bolly à par.).

Brinton (2008) relativise le caractère généralisable du chemin de grammaticalisation proposé ci-dessus et suggère pour tu vois (en angl. you see) une origine différente : c'est la constuction as you see (en fr. " comme tu vois ») (déjà présente en anglais préclassique) qui serait à l'origine de l'emploi asyndétique (i.e. sans élément segmenteur) en anglais moderne (6).

(6) Par laquelle le tant triste et piteux regard de cette povre amante t'esmeuve à pitié et compassion de ses yeux, en l'bumeur desquels amour ardent - comme tu vois - se baigne? (Litt., Préclassique, Claude de Taillemont, Discours des Champs faëz, 1553, Frantext)

L'auteur postule donc également une phase intermédiaire dans l'évolution de la construction, mais cette fois avec ellipse du as (en fr. comme) précédant le syntagme verbal, et non plus du that (en fr. que) subséquent au verbe comme on le postule 
généralement pour les autres parenthétiques (e.a. pour you know « tu sais » et I think « je pense »). Comme nous le verrons ci-dessous, la présente étude vise toutefois à nuancer cette hypothèse en proposant d'élargir le cadre d'analyse.

\subsection{Parenthétiques et évolution de la structure informationnelle}

Différents constats ont été énoncés quant à la relation qu'entretient la position syntaxique avec la fonction des marqueurs de discours dans l'organisation du discours, tant en synchronie qu'en diachronie. On postule pour un certain nombre de marqueurs (cf. e. a. Traugott 1997) un chemin évolutif au cours duquel la fonction discursive acquise serait corrélée à un mouvement de la syntaxe interne vers la périphérie syntaxique : l'emploi de départ serait celui d'un adverbial intégré au syntagme verbal (verbal adverbia), dont la fonction est propositionnelle; à un stade intermédiaire, l'unité linguistique aurait un statut d'adverbial de phrase (sentence adverb) ; au stade final de l'évolution, l'unité linguistique, se trouvant désormais en périphérie de la syntaxe, aurait une fonction proprement discursive (discourse marker) à valeur épistémique (en position initiale) ou interactionnelle (en position finale, en particulier pour le français). Chacune de ces fonctions serait donc liée à la position de l'unité linguistique au sein de l'énoncé qui l'héberge. Cette vision semble aller dans le sens des fonctions modalisatrices attribuées aux parenthétiques en initiale d'énoncé ( $f$. supra) : «subjectified elements tend to be positioned at the periphery of a constituent or clause » (Traugott, 2010, p. 41). Par ailleurs, il a été démontré que la position initiale jouait un rôle crucial dans l'organisation de l'information discursive, notamment comme lieu privilégié de mise en relief de l'information (Lambrecht 1994, 2000), de thématisation ou de topicalisation (Combettes \& Prévost 2001; Prévost 2003a; Combettes 2006).

Placer un constituant en zone initiale ne le signale pas seulement comme un élément à rattacher au contexte antérieur, comme une unité renvoyant à un référent déjà évoqué ou supposé connu, mais également comme un "point de départ » possible pour la prédication contenue dans le contexte de droite. (Combettes, 2006, p. 51-52)

Partant, nous postulons que les constructions avec tu vois (qu'elles soient parenthétiques ou intégrées syntaxiquement) jouent un rôle similaire dans l'organisation de l'information. Premièrement, il semblerait que certains emplois (observables dès le français préclassique) partagent les caractéristiques des structures présentatives à prédication seconde (Lambrecht, 2000), dont la fonction est d'introduire de nouveaux référents (ou de réactiver ceux-ci) dans le discours (cf. les « deux mains» de (7) tout en exprimant une information nouvelle sur ce référent (cf. le prédicat second «tremblantes » de (7).

(7) il mourra comme je pourray, car la premiere fois qu'il se presentera devant mes yeux, quoy qu'il en advienne, sa vie prendra fin par ces deux mains que tu voys devant toy tremblantes (Litt., Préclassique, Pierre Boaistuau, Histoires tragiques, 1559, Frantext)

Ces constructions remplissent en effet à la fois une fonction présentative, en ce qu'elles introduisent «une entité ou situation nouvelle dans un monde de discours, normalement dans le but de la rendre cognitivement accessible en vue d'une 
prédication ultérieure », et prédicative, en ce qu'elles visent à «informer l'interlocuteur d'une propriété attribuée à une entité ou une situation donnée » (Lambrecht, 2000, p. 51). Deuxièmement, d'autres types de constructions répondent à des besoins communicationnels de mise en relief d'un topique. C'est le cas notamment des segmentées à gauche (8) qui annoncent « un référent comme topique potentiel avant même qu'il ne fonctionne comme tel par la suite »(Grobet, 2002, p. 239).

(8) Va, j'en ai souffert plus que toi ; la violence que je faisois à mon coeur me punissoit assez de mon orgueil. Cet orgueil, tu vois ce qu'il est devenu : regarde-moi, je suis à tes pieds, je pleure à tes genoux (Litt., Classique, Jean-Pierre Florian, Numa Pompilius, 1786, Frantext)

À propos de ce phénomène, Lambrecht $(1994,2000)$ parle de séparation de la référence et du rôle pour désigner la dissociation qui se fait «entre la nomination du référent et son implication comme topique pronominalement marqué dans le segment suivant »(Grobet, 2002, p. 237). Nous postulons ainsi que les parenthétiques auraient un rôle à jouer dans la mise en relief de l'information et dans les processus de promotion topicale, qui s'accompagneraient d'un déplacement du poids prédicatif de l'élément verbal vois vers le constituant entrant dans sa portée argumentative (9), sur le modèle des constructions à prédication seconde.

(9) Ta foible créature implore ta clémence. Ta justice pardonne au cour qui se repent; Fais luire à ce coupable un rayon d'espérance. Tu vois mes remords infinis : S'ils ne peuvent, grand dieu! désarmer ta vengeance, Ne l'étend pas, du moins, sur ma femme et mon fils. (Théâtre, Classique, Bernard-Joseph Saurin, Béverlei, 1768, Frantext)

Sur le plan diachronique, dans le cas des constructions parenthétiques, le verbe verrait sa fonction de matrice propositionnelle affaiblie (cf. supra la notion de verbe recteur faible), permettant ainsi à la construction de se renforcer pragmatiquement et de s'autonomiser syntaxiquement au cours des siècles, en même temps que le poids prédicatif se déplacerait. Par ailleurs, comme le souligne Combettes (1998, 1999, 2006), alors que la structure à verbe second et l'ordre informationnel du moins informatif au plus informatif au sein de l'énoncé prévalent en Ancien Français ${ }^{2}$, on observe dès le Moyen Français un phénomène de recul du verbe dans la proposition en même temps qu'un enrichissement de la position préverbale ${ }^{3}$ (avec e.a. l'apparition des structures avec rhèmes secondaires et à prédication seconde). C'est durant cette période que la contrainte pesant sur la position seconde du verbe s'efface et que le principe fonctionnel fait progressivement place au principe grammatical, imposant ainsi les séquences sujetverbe. La présence d'un élément en tête n'est désormais plus incompatible avec celle du sujet.

En ancien français, l'organisation des éléments de l'énoncé selon un principe fonctionnel avait pour conséquence la présence, en tête d'énoncé, d'un élément peu informatif, qui, en outre, assurait souvent un lien avec l'énoncé précédent.

\footnotetext{
2 «Un élément 'thématique', c'est-à-dire référant à quelque chose de connu, et établissant souvent un lien avec le contexte précédent, ouvrait la proposition, et les autres éléments étaient ensuite organisés par degré croissant de valeur informative » (Prévost, 2001, p. 14-15).

${ }^{3}$ Parallèlement, les fonctions discursives coïncident de plus en plus avec la position syntaxique et l'ordre SVO se fixe progressivement.
} 
Désormais, le caractère informatif de cet élément importe peu, pourvu qu'il établisse un lien avec ce qui précède. En effet, quel que soit le principe dominant, un texte saisi dans sa continuité suppose une cohésion - plus ou moins forte - entre ses énoncés successifs. Or, souvent, celle-ci est en grande partie assurée par l'élément ouvrant l'énoncé. (Prévost, 2001, p. 17)

Dans cette étude, nous prendrons en compte certaines caractéristiques évolutives de la structure de l'information en français, mises en relation avec l'évolution de la structure syntaxique de l'énoncé. Cette approche repose donc sur une conception macro-grammaticale du changement linguistique qui serait à la fois syntaxique, sémanticopragmatique et fonctionnel.

\section{Hypothèse : constructionalisation et dé-rhématisation}

Avec l'introduction de la notion de macro-grammaticalisation ${ }^{4}$, Marchello-Nizia (2006, pp. 58, 251) appuie l'idée que les processus de grammaticalisation sont des mécanismes sous-jacents au changement linguistique, mais qu'ils sont souvent liés à d'autres types de changements linguistiques: la macro-grammaticalisation concernerait ainsi les cas où, à un niveau plus abstrait, l'apparition d'une nouvelle distinction grammaticale entraîne «un changement structurel du macro-système grammatical » ayant pour conséquence " une nouvelle hiérarchisation ou un nouveau découpage des unités de la grammaire» (Marchello-Nizia, 2006, p. 251). Cette vision nous conforte dans l'idée que la grammaticalisation ne peut pas rendre compte à elle seule de l'apparition et/ou de l'évolution des formes linguistiques, et qu'il faudrait pouvoir élargir ce cadre pour aborder certains aspects du changement. Nous pensons en outre qu'envisager l'évolution linguistique en prenant comme postulat que ce sont les formes linguistiques qui agissent sur le système, et pas l'inverse, c'est sous-estimer l'impact de la structure sémantico-pragmatique du discours sur le système langagier lui-même.

Partant de ces considérations, nous formulons l'hypothèse générale selon laquelle la constructionalisation du parenthétique tu vois serait corrélée à un processus de restructuration syntaxique et informationnelle (que nous appelons processus de dérhématisation) qui se caractérise par un recul de l'élément rhématique principal dans la structure de l'énoncé et par l'enrichissement corollaire des éléments en position initiale. Les verbes parenthétiques à l'initiale passeraient ainsi d'un statut de rhème propre à un statut de rhème secondaire (Combettes, 1998, p. 64), pour finalement perdre leur force informative en jouant un rôle moindre dans l'apport d'information ${ }^{5}$. À noter

\footnotetext{
${ }^{4}$ Cette notion s'ajoute aux changements touchant au micro-système de la langue, qui sont liés (i) à l'apparition d'une nouvelle forme dans un paradigme existant, (ii) à l'apparition d'un nouveau paradigme exprimant la même notion (ou une notion proche) déjà existante ou (iii) à l'apparition d'une nouvelle catégorie grammaticale par l'apparition d'un nouveau morphème ou paradigme (Marchello-Nizia, 2006, p. 251).

5 « $[\mathrm{D}]$ ans l'analyse de la répartition de l'information sur la ligne de la phrase, le thème désigne le constituant qui porte le degré le moins élevé de dynamisme communicatif, l'élément le moins informatif. Le rbème correspond à la partie informative de l'énoncé. Le dynamisme communicatif s'organisant de façon progressive, on pourra distinguer aussi thème secondaire et rhème secondaire » (Combettes, 1998, pp. 137-138)
} 
que par rhème secondaire, nous entendons l'élément rhématique de l'énoncé « qui apporte une information liée au rhème principal, dépendant de lui, mais ne constituant pas la partie la plus informative de l'énoncé » (Combettes, 1998, p. 64). Autrement dit, il y aurait réanalyse et recatégorisation de la construction qui verrait celle-ci migrer, selon la terminologie utilisée, de la syntaxe interne vers la syntaxe externe (« inner »- et « outersyntax »- cf. Lindström 2001 ; Lindström \& Karlsson 2005) ou de la micro-syntaxe vers la macro-syntaxe (Blanche-Benveniste 2003 ; Berrendonner 2002, 2004). Dans une perspective ouvertement héritée de la tripartition fonctionnelle de Halliday et Hasan (1976), qui distinguent les niveaux linguistiques propositionnel («ideational»), textuel (« textual») et interpersonnel (« interpersonal »), Traugott $(1982,2010)$ s'intéresse au processus de changement sémantique (« semanticization») qui voit des formes linguistiques ayant un sens non ou peu subjectivisé ("non-/less subjectivized») (au niveau propositionnel) acquérir au fil du temps un sens de plus en plus subjectivisé (« subjectivized») puis intersubjectivisé («intersubjectivized») (au niveau interpersonnel), i.e. orienté respectivement vers le locuteur/scripteur puis vers l'interlocuteur. Comme nous le verrons, ce processus d'(inter)subjectification se traduit chez Combettes en termes de passage du niveau propositionnel au niveau énonciatif (Combettes, 2006, pp. 48-49). S'inscrivant dans une approche évolutive de la structure syntaxique et informationnelle du français, c'est cette dernière terminologie qui guidera la suite de l'article.

\section{Résultats}

Avant de faire état des résultats de l'analyse proprement dite, il convient d'aborder la question des corpus utilisés. La présente étude s'appuie sur l'examen de traces de l'oral dans des écrits dit mimétiques de l'oral (Koch et Österreicher 2001), en l'occurrence des dialogues issus de textes de théâtre et de récits de fiction narrative. Les textes retenus couvrent de manière continue les périodes de français préclassique à contemporain (1550-2008) et proviennent pour la plupart de la base de données Frantext : français préclassique (1550-1660), français classique (1661-1800), français pré-moderne (1801-1940), français moderne (1941-1989) et contemporain (1989-). Les données orales en français contemporain sont issues de la base de données $V$ alibel (Dister et al. 2009). À partir de ces corpus, toutes les séquences incluant la construction tu vois avec ou sans complément postverbal (incluant ses anciennes graphies) ont été extraites de manière automatique, puis désambigüisées manuellement. Nous avons ainsi extrait 170 occurrences à la période préclassique, 492 occurrences en français classique, 1771 occurrences en français pré-moderne et 2115 occurrences en français moderne et contemporain. La première partie de l'analyse (sous 4.1.) porte sur un échantillon total de 447 occurrences, sélectionnées aléatoirement et réparties de manière proportionnelle dans chaque sous-corpus (50 occ. par type de texte et par période). Parmi ces 447 occurrences étudiées, nous porterons une attention toute particulière, dans la seconde partie consacrée aux résultats d'analyse (sous 4.2.), aux 264 occurrences de tu vois qui se trouvent en position initiale dans les textes mimétiques de l'oral.

Il est important de souligner que nous avons conscience des limites inhérentes à toute étude sur corpus en diachronie du français, en particulier quand on s'intéresse 
aux marqueurs de discours, puisque l'absence de données strictement orales avant l'époque moderne force l'examen de données qui ne sont en fait qu'une « représentation » de l'oralité (cf. Marchello-Nizia, 1999, p. 4). La nature proprement interactionnelle de tu vois (liée à la fois à la présence du pronom personnel de la $2^{\mathrm{e}}$ personne et au sémantisme du verbe) réduit toutefois l'impact de ce biais méthodologique. Dans une étude précédente, Bolly \& Degand (2009) ont ainsi montré que les constructions contenant tu vois, quelle que soit leur source textuelle (théâtre, fiction narrative ou transcription de l'oral), apparaissaient presqu'exclusivement dans des contextes immédiats qui se caractérisent par de nombreux traits de l'oralité : indicateurs de discours rapporté tels que les tirets et les guillemets, marqueurs phatiques, termes d'adresse, etc. Les corpus investigués ont donc été considérés ici comme étant suffisamment représentatifs d'une certaine forme d'oralité. Nous avons néanmoins conscience que la généralisation des résultats obtenus dans le cadre de cet article est limitée aux types de textes (eux-mêmes mimétiques de l'oral) et à l'objet linguistique qui y sont étudiés.

Plusieurs paramètres ont été considérés comme des indices révélateurs du processus de constructionalisation de tu vois. Nous montrons ci-dessous comment ceux-ci ont été opérationnalisés et quels sont les résultats qui ont émané de l'analyse sur corpus.

\subsection{Paratactisation et déplacement sémantique par abstraction}

Du point de vue de la grammaticalisation, nous avons montré ailleurs (Bolly 2010, 2011) qu'il semblait y avoir une évolution du marqueur parenthétique tu vois, depuis le français préclassique jusqu'à nos jours, qui s'apparente au processus de grammaticalisation. Cette évolution se traduit par une tendance des constructions avec tu vois au déplacement sémantique par abstraction, avec inter-subjectification. Ce déplacement sémantique est surtout marqué à partir du français moderne et contemporain (de 1941 à nos jours), puisque la fréquence d'emplois abstraits de cognition (subjectifs) (10) et d'emplois pragmatiques de ponctuants (intersubjectifs) (11) augmente fortement, alors que les emplois exprimant un constat (moins subjectifs) diminuent à la même époque (12).

(10) - Tu vois, dit-elle, bébé n'a besoin que de bien peu de chose: lui humecter les lèvres d'ean sucrée, baigner sa petite tête chaude, c'est tout ce que je puis faire. (Litt., Pré-moderne, Joseph Malègue, Augustin ou le Maître est là, 1933, Frantext)

(11) L1 oui c'est vrai mais / / enfin oui / / monais c'est c'est peut-être parce que / je veux dire / tu vois / moi depuis que je suis à l'université des gens t'en rencontres tout le temps / I$<L 3>$ oui oui - | et et eub c'est pas ça / c'est que / bon tu vois en rénové les gens / tu les rencontres mais tu les revois tous les jours [...] (Oral, Contemporain, Valibel)

(12) YVAN. Tu exagères!...

SERGE. Tu vois, il ne dit pas que j'ai tort, il dit que j'exagère, il ne dit pas que j'ai tort. (Théâtre, Contemporain, Yasmina Reza, Art, 1995, Ext. Frantext)

Quant à l'évolution syntaxique, les résultats montrent une tendance des constructions verbales incluant tu vois à la complexification jusqu'à l'époque classique, et 
ensuite à la paratactisation dès l'époque pré-moderne où la fréquence des structures asyndétiques incidentes (cf. supra 10) s'accompagne d'une baisse de fréquence de toutes les autres structures syntaxiques avec complément postverbal intégré (syntagme nominal (13), proposition infinitive (14), complétive ou interrogative (15), etc.) qui étaient jusqu'alors prédominantes. Ces résultats semblent confirmer qu'il y a bien évolution d'un emploi originellement micro-syntaxique de la construction vers un emploi macro-syntaxique du marqueur pragmatique tu vois.

(13) MATAMORE. Contemple, mon amy, contemple ce visage: Tu vois un abregé de toutes les vertus. (Théâtre, Préclassique, Pierre Corneille, L'Illusion comique, 1639, Frantext)

(14) Tu vois pleuvoir sur moy, disgrace sur disgrace (Théâtre, Préclassique, Georges de Scudéry, L'Amour tirannique, 1640, Frantext)

(15) DOROTHÉE. Mon pere estant reduit à la fin de ses jours, D'une mourante voix me tint un tel discours : Ma fille, me dit-il, tu vois que la nature Me presse d'achever ma derniere avanture. (Théâtre, Préclassique, Pichou, Les Folies de Cardenio, 1629, Frantext)

Au-delà de ces résultats, nous avons observé que l'évolution du parenthétique tu vois pouvait pourtant s'éloigner du schéma de la grammaticalisation, qu'il s'agisse de la perspective traditionnelle - qui postule une phase intermédiaire où la particule complétive que serait omise - ou l'approche plus récente de Brinton (2008) - qui suggère pour you see ("tu vois») une origine liées aux constructions parenthétiques introduites par as (" comme ») (dès l'anglais préclassique). Selon nous, il parait en effet arbitraire de rejeter de facto la possibilité que ces marqueurs puissent trouver leur origine dans une structure non subordonnante, avec par exemple un complément SN avec nom prédicatif (16) ou avec une particule complétive autre que que (17).

(16) Je lui disois : "Être éternel! $\boldsymbol{T u}$ vois les efforts de quelques-uns pour te plaire, et les tourmens que se donnent beaucoup d'autres pour t'offenser; [...]» (Litt., Classique, Joseph de Loaisel de Tréogate, Dolbreuse ou l'Homme du siècle ramené à la vérité par le sentiment de la raison, 1783, Frantext)

(17) IDOMÉNÉE. Le roi n'ignore pas ce qu'exigent les dieux! (à Sophronyme.) Tu vois si les cruels pouvoient s'expliquer mieux. Graces à leur fureur, toute erreur se dissipe [...]. (Théâtre, Classique, Prosper Jolyot de Crébillon, Idoménée, 1706, Frantext)

C'est ce constat qui nous a poussée à explorer plus avant l'évolution de la construction parenthétique en dépassant le champ strict de la grammaticalisation. Sans pour autant nier les outils de ce cadre d'analyse, sur lesquels nous nous appuyons d'ailleurs ici, nous avons voulu replacer le phénomène de l'évolution de tu vois en tenant compte à la fois du contexte évolutif en syntaxe du français et de l'évolution de la structure informationnelle (en particulier concernant les marqueurs de topicalisation en position initiale). De la grammaticalisation, nous avons ainsi retenu, entre autres, la notion de renforcement pragmatique (en pragmaticalisation) et de macro-grammaticalisation, ainsi que le rôle joué par le contexte (en particulier la position initiale) dans la migration d'entités linguistiques vers la périphérie de la syntaxe. Sachant que la position initiale joue un rôle fondamental dans l'évolution des marqueurs de discours, à la fois du 
point de vue de l'acquisition de leur fonction pragmatique, de leur autonomisation syntaxique et de leur fonction de mise en relief de l'information (cf. supra), la suite de l'étude se centrera sur les emplois de tu vois à l'initiale d'énoncé.

\subsection{Migration en périphérie, diversification syntaxique et renforcement pragmatique}

Afin de pouvoir procéder à l'analyse paramétrique des données de corpus en termes de passage du niveau propositionnel au niveau énonciatif (selon la terminologie de Combettes), il a tout d'abord fallu déterminer la position de tu vois au sein de l'énoncé qui l'hébergeait. Or, pour déterminer la position d'une unité linguistique, on ne peut pas faire l'économie de la question des frontières entre unités syntaxiques. Comme illustré dans le tableau ci-dessous, nous avons opté pour une méthode de segmentation ascendante qui parte de l'identification de l'élément constructeur au sein de l'énoncé (généralement le prédicat) vers ses éléments valenciels (constitutifs du middle field) et régis (constitutifs des initial et end-fields) (Lindström 2001; Lindström et Karlsson 2005). Ces éléments centraux entretiennent une relation de dépendance microsyntaxique avec l'élément constructeur (au sens de Blanche-Benveniste et al. 1990; Blanche-Benveniste 2003) : ils relèvent de la syntaxe interne de l'énoncé (au sens de Lindström et collaborateur).

\begin{tabular}{|c|c|c|c|c|}
\hline $\begin{array}{c}\text { Macro-syntaxe } \\
\text { Syntaxe externe }\end{array}$ & \multicolumn{3}{|c|}{$\begin{array}{c}\text { Micro-syntaxe } \\
\text { Syntaxe interne }\end{array}$} & $\begin{array}{c}\text { Macro-syntaxe } \\
\text { Syntaxe externe }\end{array}$ \\
\hline «Pre-front field» & «Initial field» & «Middle field» & "End-field» & "Post-field» \\
\hline $\begin{array}{c}\text { mais je veux dire } \\
\text { tu vois }\end{array}$ & au début & $\begin{array}{c}c c \text { / ça a l'air de } \\
\text { bien fonctionner }\end{array}$ & $\begin{array}{c}\text { comme système parce } \\
\text { que / tout le monde a } \\
\text { l'air gentil }\end{array}$ & etcaetera \\
\hline
\end{tabular}

Tableau 1. Syntaxe interne et externe d'un énoncé (26, cf. infra)

Il s'agissait ensuite d'identifier les éléments associés au niveau de la macro-syntaxe (au sens de Blanche-Benveniste et collaborateurs), i.e. relevant de la syntaxe externe et situés en périphérie gauche (pre-front field) ou droite (post-field) (au sens de Lindström et collaborateur) par rapport à l'ilot rectionnel. Une fois cette structure mise au jour pour les énoncés étudiés, la position de la construction tu vois a été opérationnalisée.

\subsubsection{Position syntaxique}

Plusieurs catégories ont été distinguées pour caractériser la position de tu vois au sein de l'énoncé qui l'hébergeait :

(i) la position initiale stricte, où tu vois se situe à la frontière gauche de l'énoncé qui le contient; soit voir est le verbe principal d'une unité syntaxique de rang supérieur (i.e. la clause) (Ex. 18), soit tu vois est syntaxiquement autonome (10); 
(18) LA PETITE POLYXÈNE : Ob! ne rien sentir! Je préférerais rester et ne plus jamais rien sentir...

HÉLÈNE : $\boldsymbol{T u}$ vois comme tu t'exprimes mal! Pour que je parte, au contraire, il faudrait que je ne vous aime pas. Tu préferes que je ne t'aime pas? (Théâtre, Prémoderne, Jean Giraudoux, La Guerre de Troie n'aura pas lieu, 1935, Frantext)

(ii) la position quasi initiale, où tu vois est précédé d'un élément macro-discursif ou extrapropositionnel (par ex. un marqueur pragmatique ou une interpellation) (19) ;

(19) Le Sous-Préfet, qui est à l'extrême gauche du piano, à sa femme qui est près de lui. - Ehb! Bien, tu vois, ma chère amie, ce sont ces petites choses-là qu'il faut connaître! Ce sont des riens !... mais c'est à ces riens-là qu'on reconnaît la parisienne. (Théâtre, Pré-moderne, Georges Feydeau, La Dame de chez Maxim, 1914, Frantext)

(iii) la position médiane, où la construction est obligatoirement intégrée micro-syntaxiquement et est au minimum précédée (et parfois suivie) d'un élément de rection (par ex. une conjonction de subordination ou un complément circonstanciel antéposé) (20) ;

(20) - Qu'est-ce que c'est, ça? - C'est du vieux fer ; cet endroit qui luit, tu vois, sur les trous, c'est un endroit où le vieux fer bien dur a été usé par la peau de la bouche. (Litt., Prémoderne, Jean Giono, Un de Baumugnes, 1929, Frantext)

(iv) la position quasi finale, où tu vois n'est pas intégré micro-syntaxiquement et est suivi d'un élément macro-discursif ou extra-propositionnel (21);

(21) HECTOR : Et c'est tout?

ULYSSE : C'est tout.

OIAX : Ce n'est pas long, tu vois, Hector?

(Théâtre, Pré-moderne, Jean Giraudoux, La Guerre de Troie n'aura pas lieu, 1935, Frantext)

(v) la position finale stricte, où tu vois n'est pas intégré micro-syntaxiquement et se situe à la frontière droite de l'énoncé (22);

(22) - Tu m'en veux, pour bier? demanda-t-elle.

- Comme si je pouvais t'en vouloir, à toi?

- Je suis venue dès que j'ai pu, tu vois. Et j'ai couru, tu sais.

(Litt., Moderne, Bernard Clavel, Le Coeur des vivants, 1964, Frantext)

(vi) la position transitionnelle, où tu vois est à la frontière de deux unités de discours autonomes et possède une portée sémantico-pragmatique ambigüe (localisable soit sur le segment à gauche, soit sur le segment à droite, soit sur les deux, sans que l'on puisse décider de l'une ou l'autre possibilité) (23).

(23) L1 ob ça là j'étais énervée parce que en fait le mec ç/ c'est un de mes copains quoi / [...] déjà il était paralysé et ci et là alors que c'est pas vrai quoi mais $i$-z- exagèrent tout le temps / alors là je l'ai mal pris / à ce moment-là j'ai mal réagi tu vois i font chier pour finir hein / mais maintenant je m'en fous (Oral, Contemporain, Valibel)

Les résultats obtenus ( $c f$. Tableau 2) qui, pour répondre au critère de représentativité des corpus, ne concernent ici que les textes mimétiques de l'oral, semblent confirmer que les structures incluant tu vois évoluent vers une position de plus en plus périphérique. 


\begin{tabular}{|l|l|l|l|l|l|}
\hline \multicolumn{1}{|c|}{ tu vois } & $\begin{array}{l}\text { Préclassique } \\
1550-1660\end{array}$ & $\begin{array}{l}\text { Classique } \\
1661-1800\end{array}$ & $\begin{array}{l}\text { Pré-moderne } \\
1801-1940\end{array}$ & $\begin{array}{l}\text { Mod./Contemp. } \\
1941-\end{array}$ & Total \\
\hline Initiale & $46(47,4 \%)$ & $69(69 \%)$ & $71(71 \%)$ & $78(78 \%)$ & 264 \\
\hline Médiane & $51(52,6 \%)$ & $31(31 \%)$ & $20(20 \%)$ & $19(24 \%)$ & 121 \\
\hline Finale & $0(0 \%)$ & $0(0 \%)$ & $9(9 \%)$ & $3(3 \%)$ & 12 \\
\hline Total & $97(100 \%)$ & $100(100 \%)$ & $100(100 \%)$ & $150(100 \%)$ & 397 \\
\hline
\end{tabular}

Tableau 2. Evolution de la position syntaxique de tu vois

Partagé entre la position initiale de tu vois (en ce compris les initiaux stricts, les quasi initiaux et les transitionnels) et la position médiane à l'époque préclassique (environ un cas sur deux dans l'une et l'autre position), le nombre d'occurrences en position initiale augmente progressivement au cours de temps (plus de trois quarts des cas à l'époque moderne et contemporaine). Parallèlement, les cas en position médiane diminuent dès le français classique (passant de un cas sur deux à moins de un cas sur trois). Quant aux emplois en finale, ils sont peu fréquents et concernent à peine un cas sur dix à l'époque moderne et contemporaine.

Il est intéressant de noter que si l'on considère la distinction en types de textes, les textes de théâtre montrent une préférence pour les emplois à l'initiale dès le français préclassique (deux cas sur trois environ), alors que les textes littéraires de la même période contiennent davantage de cas en position médiane (deux cas sur trois environ). L'évolution de la fréquence au cours des siècles suit cependant la tendance générale qui voit une augmentation des cas à l'initiale et une diminution des cas en position médiane, pour les deux types de textes, avec une évolution plus nette dans le corpus littéraire qui passe d'un cas sur trois en préclassique à plus de deux cas sur trois en français moderne et contemporain. Pour l'oral, la proportion est d'un peu moins de deux cas sur trois en position initiale, contre un tiers en position médiane et seulement un cas sur seize en position finale.

Ces résultats tendent à montrer que la construction tu vois subit un déplacement sémantico-pragmatique et une paratactisation progressive, corrélés à un changement de fonction et de position dans la structure de l'énoncé qui l'héberge.

\subsubsection{Diversification syntaxique de la portée}

Pour localiser la portée de tu vois, nous avons appliqué des tests de transformation syntaxique permettant de déterminer quel était l'objet du procès de voir, tels que le dispositif du pseudo-clivage (Ce qui est vu/ constatél compris/ etc., c'est...), la transformation interrogative (Qu'est-ce qui est (supposé être) vu/ constatél compris/ etc. par l'interlocuteur?) et le test d'équivalence avec une proforme (le, la, les, en, cela). Ces tests ont été appliqués aux occurrences prises dans leur contexte d'énonciation, tenant compte de chacune des acceptions du verbe observées en corpus (perceptuelle, de témoignage, expérientielle, de constat, de représentation cognitive et de compréhension). Par ailleurs, ont été retenus comme étant des indices d'intégration micro-syntaxique de la portée : (i) le marquage morphosyntaxique de la relation par un ligateur (que, combien, comment, lequel, etc.) ou par une structure subordonnante (infinitive, participial, etc.) et/ou (ii) la présence d'adverbiaux ou de circonstants entrant dans la rection du verbe 
voir (par ex. bien, facilement, etc.). Dans les exemples qui suivent, les portées seront délimitées par des crochets.

Si l'on en croit Combettes (2006), le passage au niveau énonciatif se traduirait par la diversité syntaxique grandissante des types de constituants pouvant entrer dans la portée de l'unité se trouvant à l'initiale. Un examen des types de structure syntaxique entrant dans la portée de tu vois (incluant les initiaux, les quasi initiaux et les transitionnels) donne un premier aperçu de la répartition des différentes structures au cours des siècles ( $c$. Tableau 3). Ces résultats montrent que les cas dont la portée est constituée d'un syntagme nominal régi par le verbe voir $(9,13,16)$ sont très fréquents à l'époque pré-classique et classique (avec plus d'un cas sur trois). Ils apparaissent ensuite dans une proportion nettement inférieure, avec environ un cas sur huit en français pré-moderne et moins d'un cas sur dix en français moderne et contemporain.

\begin{tabular}{|l|c|c|c|c|c|}
\hline tu vois initial & $\begin{array}{c}\text { Préclassique } \\
1550-1660\end{array}$ & $\begin{array}{c}\text { Classique } \\
1661-1800\end{array}$ & $\begin{array}{c}\text { Pré-moderne } \\
1801-1940\end{array}$ & $\begin{array}{c}\text { Mod./Contemp. } \\
1941-\end{array}$ & Total \\
\hline Synt. nominal & $16(34,8 \%)$ & $24(34,8 \%)$ & $9(12,7 \%)$ & $6(7,7 \%)$ & 55 \\
\hline Prop. infin. & $3(6,5 \%)$ & $1(1,5 \%)$ & 0 & 0 & 4 \\
\hline Prop. syndétique & $25(54,4 \%)$ & $39(56,5 \%)$ & $29(40,8 \%)$ & $14(18 \%)$ & 107 \\
\hline Asyndétique & $2(4,3 \%)$ & $5(7,2 \%)$ & $33(46,5 \%)$ & $58(74,3 \%)$ & 98 \\
\hline $\begin{array}{c}\text { - Prop. indép. } \\
\begin{array}{c}\text { - Extralingu., } \\
\text { implicite }\end{array}\end{array}$ & 1 & 2 & 26 & 38 & 67 \\
\hline $\begin{array}{c}\text { - Mixte, } \\
\text { ambigu }\end{array}$ & 0 & 3 & 5 & 15 & 24 \\
\hline Total & $46(100 \%)$ & $69(100 \%)$ & $71(100 \%)$ & $78(100 \%)$ & 264 \\
\hline
\end{tabular}

Tableau 3. Évolution de la nature syntaxique de la portée de tu vois

Parallèlement, les constructions syndétiques, qui se caractérisent par la présence d'un élément ligateur (que, qui, si, comme, combien, -quel ou -où) entre le verbe voir et la proposition qu'il introduit (15), restent majoritaires jusqu'à l'époque pré-moderne, où elles concernent encore presqu'un cas sur deux (18). Elles subissent ensuite un net recul à l'époque moderne, avec moins d'un cas sur cinq.

Suivant un mouvement inversement proportionnel, on voit de plus en plus de constructions asyndétiques apparaître à l'initiale d'énoncé : partant de moins d'un cas sur vingt en français préclassique (24), celles-ci représentent près de trois quarts des emplois de tu vois à l'époque moderne et contemporaine (25).

(24) Ayes pour Dieu compassion de nous,

Tu vois [helas! nous courbons les genoux

Devant tes pieds.]

(Théâtre, Préclass., Jean de La Taille, La Famine ou les Gabéonites, 1573, Frantext)

(25) GERMAIN - Elle, c'est différent. Mes discours rationnels ne s'adressent pas à Ludivine, ni à Benjamin. Mais tu vois, [les Martiens, ça laisse certains adultes indifférents, ça en fait rire d'autres, et ça rend dingue le reste, c'est-à-dire la majorité.] (Théâtre, Contemporain, Philippe Bastin, E.T. à Houte-si-Plout, 2007, Ext. Frantext) 
Les asyndétiques sont constituées essentiellement de structures propositionnelles (i.e. constituées d'un ou plusieurs énoncés) jusqu'à l'époque pré-moderne incluse, où elles représentent quatre cas sur cinq. Ces emplois issus de textes mimétiques de l'oral (25, ci-dessus) sont très représentatifs de l'oral d'aujourd'hui (26, ci-dessous).

(26) L1 mais je veux dire tu vois [au début ça / ça a l'air de bien fonctionner comme système parce que / tout le monde a l'air gentil etcaetera] et puis / tu te rends compte après deux mois qu'i-y-a des gens qui commencent à se nier / etcaetera je sais pas (Oral, Contemporain, Valibel)

La proportion des structures propositionnelles au sein des asyndétiques diminue ensuite légèrement (deux asyndétiques sur trois) à l'époque moderne. La diversification des structures asyndétiques s'accompagne à cette époque d'une augmentation du nombre d'emplois dont la portée est extralinguistique, c'est-à-dire contextuelle, non verbale ou implicite. On passe en effet de moins d'une structure asyndétique sur dix à portée extralinguistique, en français pré-moderne, à plus d'une structure asyndétique sur quatre en français moderne et contemporain (27).

(27) Ca c'est pour se protéger du manvais sort. Tu la mets autour de ton cou et quand t'es en danger tu replies sur ton pouce les doigts du milieu de la main droite - tu vois, Comme ca + GESTE] - et tu fais les cornes au diable. (Litt., Contemporain, Fanny Seguin, L'Arme à gauche, 1990, Frantext)

Les constructions asyndétiques avec tu vois peuvent aussi, bien que plus rarement, être constituées d'un syntagme nominal non régi (28) ou d'un fragment textuel syntaxiquement incomplet (29). Elles se rapprochent dans ce cas des constructions dont la portée est implicite et ont par conséquent été catégorisées comme telles, bien qu'au moins une partie de leur contenu soit explicite (ici : «Mes précautions »).

(28) Jeanne. On vient!

Paul. Fuyons! Comme on dit dans les tragédies.

Jeanne. Vite, vite! ...

Paul. Tu vois, hein? [Mes précautions.]

Jeanne. Déjà! Quel malheur!

(Théâtre, Pré-moderne, Édouard Pailleron, Le Monde où l'on s'ennuie, 1968, Frantext)

(29) - Eh bien, me dit A, voilà ce que je voulais. Tu vois : [dès que tu te donnes un peu de peine... - - Ne m'agace pas, lui dis-je. C'est assez. dur d'être un homme, on de l'avoir été. N'abuse pas de tes pouvoirs. (Litt., Contemporain, Jean d'Ormesson, La Douane de mer, 1993, Frantext)

Une catégorie regroupe en outre les cas ambigus ou mixtes, dont la portée comprenait plusieurs éléments de nature syntaxique différente ou donnait lieu à plusieurs interprétations possibles (30)

(30) Doucement il caressait le grain de la pierre : "C'est ton fils, Maman. [Je ne t'ai pas laissée], tu vois. [Je suis là]. » (Litt., Contemporain, Françoise Chandernagor, L'Enfant des Lumières, 1995, Frantext) 
De manière générale, ce sont les structures où tu vois introduit une proposition, un énoncé ou un fragment textuel (qu'elles soient syndétiques ou asyndétiques) qui sont les plus fréquentes jusqu'à l'époque pré-moderne. Mais leur fréquence diminue ensuite à l'époque moderne où de nouvelles constructions à portée extralinguistique, en particulier dans les énoncés asyndétiques, commencent à s'imposer. Ces résultats pour les emplois de tu vois à l'initiale vont donc dans le même sens que les résultats observés pour l'ensemble des constructions, quelle que soit leur position syntaxique (cf. supra, en 4.1.), à savoir une paratactisation des constructions accompagnée d'une évolution vers des emplois de plus en plus intersubjectifs.

\subsubsection{Renforcement pragmatique}

Certains contextes sont propices au renforcement pragmatique et, partant, à l'acquisition d'une fonction discursive. C'est ainsi que la co-apparition de tu vois avec d'autres marqueurs ou particules de discours (par ex. mais tu vois, eh bien tu vois, hein donc eub tu vois) semble être un bon indicateur du fonctionnement pragmatique de tu vois à l'initiale d'énoncé (cf. 26 transcrit de l'oral, supra). Déjà en français préclassique, il est courant que tu vois apparaisse précédé d'une autre entité linguistique syntaxiquement non intégrée (c'est ce que nous avons appelés les quasi initiaux). Parmi l'ensemble des occurrences de tu vois à cette période, on compte par exemple un quart environ d'emplois en position initiale stricte (31), pour un cinquième en position quasi initiale (32).

(31) Le Comte [...] luy dist: «Tu voy, ma fille, mon inconsiderée et simple promesse et l'effrené vouloir d'un Roy passionné, auquel j'ay respondu qu'il estoit en ma puissance de te prier, mais non de te forcer. [...] » (Litt., Préclassique, Pierre Boaistuau, Histoires tragiques, 1559, Frantext)

(32) Il avoit une prebende à Chartres, laquelle il laissa pour se marier avec une belle fille, à laquelle, au matin de la premiere nuict de ses nopces, il dit : "eh bien, ma mie, tu vois comme je t'ayme, d'avoir laissé ma prebende pour t'avoir ! [...] » (Litt., Préclassique, Béroalde de Verville, Le Moyen de parvenir, 1610, Frantext)

Parmi l'ensemble des emplois qui ont été considérés comme étant initiaux (regroupant les initiaux stricts, les quasi initiaux et les transitionnels, cf. supra), on remarque que la proportion de tu vois en cooccurrence avec d'autres marqueurs de discours (souligné dans l'Ex. 33) reste, malgré une légère baisse de fréquence, relativement stable au cours des siècles : on compte un cas sur quatre en français préclassique, puis respectivement un cas sur six, un cas sur cinq et un cas sur sept en français classique, pré-moderne et moderne/contemporain (33).

(33) - Qu'est-ce que vous foutez? gronda Fayolle d'une voix manvaise.

- Calme-toi, l'ami!

- Ben tu vois, expliqua Paradis, on ramasse les cuirasses, c'est la consigne. Faut rien laisser derrière nous. (Litt., Contemporain, Patrick Rambaud, La Bataille, 1997, Frantext)

Notons que quand le nombre de cas effectifs était suffisant, nous avons effectué des tests de corrélation permettant de mettre en relation plusieurs paramètres d'analyse. Les résultats statistiques ont été considérés comme significatifs quand le coefficient de contingence (p) était inférieur ou égal à 0,05 . Les résultats statistiquement non significatifs sont marqués par les initiales (NS). Ici, les résultats statistiques non 
significatifs $(\rho=-0,087, \mathrm{p}>0,1(\mathrm{NS}))^{6}$ tendent à montrer que le contexte d'apparition des tu vois initiaux, en termes de co-apparition avec d'autres marqueurs, reste stable au cours des siècles. La différence statistique n'est donc pas suffisamment significative pour qu'on puisse établir un lien de corrélation entre la co-apparition de tu vois avec d'autres marqueurs discursifs à l'initiale et son hypothétique renforcement pragmatique au cours des siècles.

Nous avons vu par ailleurs que le renforcement pragmatique pouvait se traduire par la présence, dans l'environnement immédiat de tu vois, de marques énonciatives explicites de l'implication, par le locuteur, de l'interlocuteur dans l'acte d'énonciation:

(i) structures impératives ou interrogatives, indiquées notamment par l'emploi du mode impératif (10), par la ponctuation à l'écrit (18 et 21) ou les particules phatiques à l'oral (34);

(34) L1 [...] et j'ai j'avais en quelque chose comme quarante-quatre ou quarante-cinq pour cent (LO ab oui) L1 alors euh avec des zéros partout hein tu vois le genre bein (Oral, Contemporain, Valibel)

(ii) présence de termes d'adresse dans l'environnement proche ou immédiat de th vois (cf. «mon amy» dans 13, «être éternel » dans l'Ex. 16, « ma chère amie » dans 19, «Hector» dans 21, «ma fille» dans 31 et « ma mie » dans 32).

Or, ce que les résultats d'analyse montrent, c'est que si le contexte est bien caractérisé par un certain nombre de marques énonciatives explicites, il n'y a cependant pas de renforcement pragmatique contextuel à proprement parler. Au contraire, il ressort de l'analyse des données mimétiques de l'oral une tendance significative $(\rho=-0,180, p<0,005)$ appuyant le mouvement inverse. C'est ainsi que les occurrences qui se caractérisent par un certain détachement de l'interlocuteur augmentent progressivement : en français préclassique, elles couvrent un peu plus de la moitié des cas, pour finalement concerner plus de quatre cas sur cinq en français moderne et contemporain. Inversement, les emplois marqués explicitement par une forte implication de l'interlocuteur diminuent au cours des siècles (cf. Graphique 1) : couvrant moins de la moitié des cas à l'initiale en français préclassique (43,5\%), elles ne concernent plus qu'un cas sur cinq en français actuel (19,2\%).

\footnotetext{
${ }^{6}$ Le test de corrélation de rang avec le $\rho$ (rho) de Spearman permet de mesurer l'influence réciproque de plusieurs variables dont l'une au moins est scalaire ou ordinale (ici, la périodisation).
} 


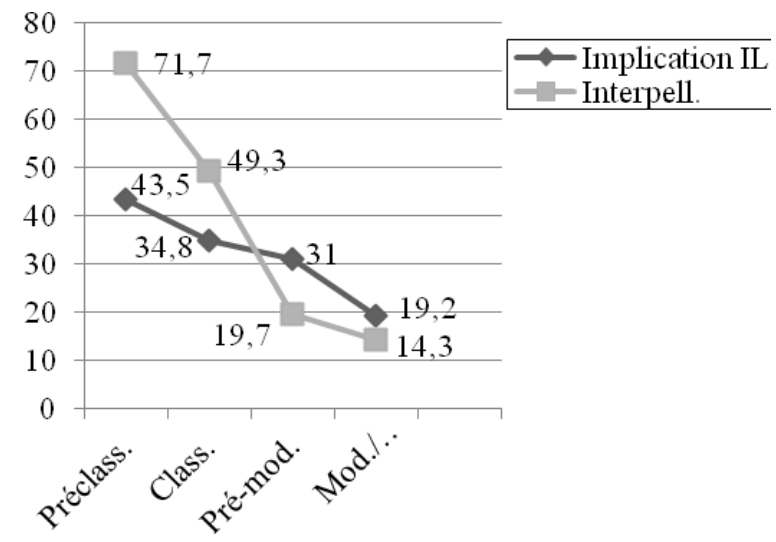

Graphique 1. Contexte énonciatif : évolution de l'implication de l'interlocuteur

De même, la fréquence des contextes interpellatifs marqués par la présence d'un terme d'adresse dans le contexte proche ou immédiat de tu vois tend à diminuer de manière nettement significative $(\rho=-0,438, p=0,000)$ au cours des siècles, passant de $71,7 \%$ de cas en français préclassique à seulement $14,3 \%$ dans les textes en français moderne et contemporain (cf. Graphique 1). Plutôt que d'assister à un renforcement pragmatique du contexte d'apparition de tu vois (auquel on s'attendait initialement), on assiste donc en quelque sorte à un affaiblissement du marquage pragmatique de l'environnement immédiat de la construction. Ce qui ne veut pas nécessairement dire que la construction en elle-même perd de sa force pragmatique, puisque nous avons mis en évidence une augmentation des emplois de plus en plus abstraits, voire même intersubjectifs, de la construction au cours des siècles.

\section{Conclusion}

Dans cette étude, nous avons choisi de désigner le processus évolutif mis au jour pour tu vois par le terme de constructionalisation, reconnaissant ainsi le rôle crucial joué par le contexte linguistique et par la collocabilité dans l'évolution de certaines unités complexes, telles que les constructions parenthétiques. D’une part, les résultats ont montré une tendance à la complexification et à la paratactisation des constructions incluant tu vois, avec un déplacement sémantique par abstraction, pour toutes les constructions étudiées quelle que soit leur position syntaxique au sein de l'énoncé qui les héberge. Ces résultats confortent lidée d'une grammaticalisation (ou pragmaticalisation) à l'œuvre pour tu vois. Allant plus loin, nous nous sommes posé la question de savoir si cette évolution pouvait être liée à un changement plus global dans la manière de structurer l'information (i .e. un type de macro-grammaticalisation). C'est ainsi que nous avons proposé d'envisager l'évolution de la construction en posant une hypothèse fonctionnelle fondée sur l'idée d'un processus de dé-rhématisation de la construction tu vois à l'initiale. Partant du postulat que la position à l'initiale joue un rôle prépondérant dans l'organisation discursive, nous avons constaté que les constructions à l'initiale (qu'elles soient syndétiques ou non) voyaient leur portée syntaxique se diversifier, ce 
qui va dans le sens d'une pragmaticalisation de tu vois agissant de plus en plus au niveau macrosyntaxique du langage. Par ailleurs, nous avons vu que les emplois à l'initiale se caractérisaient par la dimension proprement énonciative de leur environnement immédiat, explicitement orientée vers l'interlocuteur, dès le français préclassique et jusqu'à l'époque contemporaine. Si le contexte énonciatif semble être effectivement propice à la pragmaticalisation de tu vois, il n'en reste donc pas moins que le contexte se défait progressivement de tout marquage explicite de cette fonction pragmatique. Est-ce à dire que ces marques ne sont désormais plus nécessaires pour appuyer la force pragmatique de l'énoncé ? La construction en elle-même serait-elle devenue suffisamment puissante pour que l'interlocuteur se sente impliqué dans le discours d'autrui sans que ce dernier ne doive user d'autres marques linguistiques? Ou bien serait-ce au contraire la perte de force interpersonnelle de la construction elle-même qui rendrait possible l'émergence des emplois à fonction de ponctuants (Dehé et Wichmann 2010a, 2010b), d'atténuation (Schneider 2007) ou évidentielle (Bolly 2010, 2011) ? Ce sont là des hypothèses fortes, qu'il nous semblerait intéressant de pousser plus avant dans de futures recherches. Suivant la tripartition de Dehé et Wichmann (2010a, 2010b), nous pensons en outre que tu vois acquerrait progressivement un statut macrosyntaxique lui conférant la possibilité de remplir en français parlé, à un stade plus ou moins avancé du processus, une fonction d'atténuation, de marqueur évidentiel, de mise en relief de l'information et/ou de marqueur phatique. Il n'en reste pas moins que la manière dont ces différentes fonctions interagissent reste à explorer, tenant (idéalement) compte des différents plans d'analyse en jeu, qu'ils soient formel, syntaxique, prosodique, sémantique, pragmatique ou informationnel.

En conclusion, des analyses qualitatives exprimées en termes de structuration (tenant compte des enchaînements topique-commentaire) et/ou de saillance informationnelle (tenant compte du degré d'accessibilité des représentations du référent) permettraient certainement de vérifier de manière plus solide l'hypothèse de départ, qui a été testée ici via l'opérationnalisation des critères d'autonomisation syntaxique, de déplacement sémantique et de renforcement pragmatique. Étendre l'analyse aux occurrences de $t u$ vois en position non strictement initiale et couvrir des périodes antérieures au $16^{\mathrm{e}}$ siècle donnerait également la possibilité de vérifier si le changement postulé est généralisable de ce point de vue. En bref, la présente étude, si elle n’a pas véritablement permis d'asseoir la validité de l'hypothèse de départ, ne la contredit cependant pas non plus tout à fait. Elle présente par ailleurs, bien que de manière programmatique, de nouvelles pistes de réflexion sur le changement langagier.

\section{Remerciements}

L'auteur est chargée de recherches du Fonds de la Recherche Scientifique (F.R.S.-FNRS) de Belgique. Cet article est le fruit de recherches menées en collaboration avec l'Université de Neuchâtel (Suisse) qui ont été rendues possibles notamment grâce à un subside du Fonds Spécial de Recherche (FSR) de l'Université catholique de Louvain et à un crédit pour bref séjour du F.R.S.-FNRS. Cette étude s'intègre par ailleurs dans le Pôle d'Attraction Interuniversitaire "Grammaticalization and (inter)Subjectification » (contrat PAI P6/44) financé par le gouvernement fédéral belge. 


\section{RÉFÉRENCES BIBLIOGRAPHIQUES}

Andersen, H.L. (1997), Propositions parenthétiques et subordination en français parlé. Thèse de doctorat de l'université de Copenhague.

ANDERSEN, H.L. (2007), « Marqueurs discursifs propositionnels », Langue française, $n^{\circ}$ 154, pp. 13-28.

Ajjmer, K. (2007), "The interface between discourse and grammar: The fact is that ", in Celle A. \& Huart R. (éds), Connectives as Discourse Landmarks, Amsterdam-Philadephia, John Benjamins, pp. 31-46.

Auer, P. (1996), «The pre-front field in SPoken German and its relevance as a grammaticalization position », in Pragmatics, nº/3, pp. 295-323.

BÉGUELIN, M.-J. (2009), «From the confession of ignorance to the indefinite : what impact for a theory of grammaticalization ?", in Rossari C. et alii (éds), Facts, Approaches, Theorical Issues. Studies in Pragmatics, pp. 35-64.

BÉGuelin, M.-J. (2010), «Le statut des identités diachroniques dans la théorie saussurienne: une critique anticipée du concept de grammaticalisation », in Bronckart, J.-P., Bota C. \& Bulea E. (éds), Le projet de Saussure, Genève, Droz, pp. 237-268.

Berrendonner, A. (2002), "Morpho-syntaxe, pragma-syntaxe et ambivalences sémantiques », in Andersen, H.L. \& Nølke N. (éds), Macro-syntaxe et macro-sémantique (Actes du colloque d'Aarbus, 20-22 mai 2001), Berne, Peter Lang, pp. 23-41.

Berrendonner, A. (2004), "Grammaire de l'écrit vs grammaire de l'oral : le jeu des composantes micro- et macro-syntaxiques ", in Rabatel, A. (éds), Interactions orales en contexte didactique, Lyon : Presses Universitaires de Lyon, pp. 249-264.

BlanCHE-BenVEnisTE, C. (1989), «Constructions verbales en 'incise' et rection faible des verbes», Recherches sur le français parlé, $\mathrm{n}^{\circ} 9$, pp. 53-73.

BLANCHE-BENVENISTE, C. (2003), «Le recouvrement de la syntaxe et de la macro-syntaxe », in A. Scarano (dir.), Macro-syntaxe et pragmatique. L'analyse linguistique de l'oral, Roma, Bulzoni, pp. 53-75.

Blanche-Benveniste, C., Bilger, M., Rouget, C., Van den Eynde, K. \& Mertens, P. (1990), Le français parlé. Études grammaticales, Paris, Éditions du CNRS.

Blanche-Benveniste, C. \& WiLlems, D. (2007), «Un nouveau regard sur les verbes 'faibles'», Bulletin de la Société de linguistique de Paris, $\mathrm{n}^{\circ} 102 / 1$, pp. 217-254.

Benveniste, É. (1966), Problèmes de linguistique générale I, Paris, Gallimard.

Bolly, C. (2010), «Pragmaticalisation du marqueur discursif tu vois. De la perception à l'évidence et de l'évidence au discours », in Neveu, F., Durand, J., Klingler, T., Prévost, S. \& Muni-Toké, V. (éds), Actes du CMLF 2010 (2e Congrès Mondial de Linguistique Française, 12-15 juillet 2010, New Orleans, Etats-Unis).

Bolly, C. (2011), «Du verbe de perception visuelle au marqueur parenthétique tu vois. Grammaticalisation et changement linguistique », Journal of French Language Studies, n²2/3, pp. 1-22. 
BOLly, C. (à paraitre), «Flou phraséologique, quasi-grammaticalisation et pseudo marqueurs de discours. Un no man's land entre syntaxe et discours ? ", in V. Conti, G. Corminbœuf et L.A. Jonhsen (éds), Entre syntaxe et discours, LINX.

Bolly, C. \& DEGAND, L. (2009), «Frequency effects on the evolution of discourse markers in spoken vs. written French». Communication orale présentée à la Corpus Linguistics Conference (21-23 juillet 2009, University of Liverpool, Royaume-Uni).

BRInTON, L.J. (1996), Pragmatic Markers in English: Grammaticalization and Discourse Functions, Berlin, Mouton de Gruyter.

BRInTON, L.J. (2008), The Comment Clause in English. Syntactic Origins and Pragmatic Development, Cambridge, CUP, Coll. Studies in English Language.

Bybee, J. (2003), "Mechanisms of change in grammaticalization: The role of frequency », in Joseph, B.D. \& Janda, R.D. (eds), The Handbook of Historical Linguistics, Oxford, Blackwell, pp. 604-623.

Bybee, J. (2006), «From usage to grammar: The mind's response to repetition », Language, n $82 / 4$, pp. 711-734.

BYbeE, J. \& Torres Cacoullos, R. (2009), "The role of prefabs in grammaticization: How the particular and the general interact in language change ", in Corrigan, R., Moravcsik, E., Ouali, H. \& Wheatley, K. (eds), Formulaic Language (Volume 1: Distribution and historical change), Amsterdam-Philadelphia, John Benjamins, pp. 187-217.

Charolles, M. (2003), «De la topicalité des adverbiaux détachés en tête de phrase », Travaux de Linguistique, $\mathrm{n}^{\circ} 47$, pp. 11-51.

COMBETTES, B. (1998), Les constructions détachées en français, Paris, Ophrys.

COMbetTes, B. (1999), «Thématisation et topicalisation : leur rôle respectif dans l'évolution du français », in Guimier, C. (dir.), La thématisation dans les langues. Actes du colloque de Caen, 911 octobre 1997, Bern-Berlin, Peter Lang, pp. 231-245.

CombetTes, B. (2006), « Du niveau textuel au niveau énonciatif dans la grammaticalisation : le rôle du contexte ", Langue française, $\mathrm{n}^{\circ} 149 / 1$, pp. 48-60.

Combettes, B. \& Prévost, S. (2001), «Évolution des marqueurs de topicalisation », Cabiers de Praxématique, $\mathrm{n}^{\circ} 37$, pp. 103-124.

CORnUlier, B. de (1978), "L'incise, la classe des verbes parenthétiques et le signe mimique », Cabier de linguistique, $\mathrm{n}^{\circ} 8$, pp. 53-95.

Dehé, N. \& Wichmann, A. (2010a), «Sentence-initial I think (that) and I believe (that): Prosodic evidence for use as main clause, comment clause and discourse marker», Studies in Language, $\mathrm{n}^{\circ} 34 / 1$, pp. 36-74

DehÉ, N. \& Wichmann, A. (2010b), «The multifunctionality of epistemic parentheticals in discourse : Prosodic cues to the semantic-pragmatic boundary », Functions of Language, nº17/1, pp. 1-28.

Diewald, G. (2006), «Context types in grammaticalization as constructions », in Schönefeld, D. (dir.), Constructions. Special Volume 1 : Constructions all over - case studies and theoretical implications. [http://www.constructions-online.de/articles/specvol1/] 
Catherine Bolly

Diewald, G. (2010), "On some problem areas in grammaticalization theory », in Stathi, K., Gehweiler, E. \& König, E. (éds), Grammaticalization. Current views and issues (Studies in Language Companion Series 119), Amsterdam-Philadelphia, John Benjamins, pp. 17-50.

Dister, A., Francard, M., Hambye, P. \& Simon, A.C. (2009), «Du corpus à la banque de données. Du son, des textes et des métadonnées. L'évolution de banque de données textuelles orales VALIBEL (1989-2009) », Cabiers de Linguistique, n³3/2, pp. 113-129.

Dostie, G. (2004), Pragmaticalisation et marqueurs discursifs. Analyse sémantique et traitement lexicographique. Bruxelles, De Boeck-Duculot, Coll. Champs linguistiques.

Erman, B. \& Kotsinas, U.-B. (1993), «Pragmaticalization : the case of 'ba' and 'you know'», Studier i modern språkvetenskap, $\mathrm{n}^{\circ} 10$, pp. 76-93.

FranÇOIS, J. \& LEGAllois, D. (dir.) (2006), Autour des grammaires de constructions et de patterns, Cahiers du CRISCO 21.

GoldBerg, A. (1995), Constructions : A construction grammar account of argument structure, Chicago : University of Chicago Press.

GonZÁLEZ, M. (2008), « Pragmatic markers and discourse coherence relations in English and Catalan oral narrative », Discourse Studies, nº7/1, pp. 53-86.

HANSEN, M.-B. (1998), The function of discourse particles, Amsterdam-Philadelphia : John Benjamins.

Haspelmath, M. (2004), «On directionality in language change with particular reference to grammaticalization ", in Fischer, O., Norde, M. \& Perridon, H. (eds), Up and Down the Cline : The Nature of Grammaticalization, Amsterdam : John Benjamins, pp. 17-44.

HeINE, B. (2002), «On the role of context in grammaticalization», in Wischer, I. \& Diewald, G., New Reflections on Grammaticalization (Typological studies in language 49), AmsterdamPhiladelphia, John Benjamins, pp. 83-101.

Hopper, P.J. (1987), « Emergent Grammar », Berkeley Linguistics Society, n¹3, pp. 139-157.

Hopper, P.J. (1991), «On some principles of grammaticalization », in Traugott, E.C. \& Heine, B. (eds), Approaches to Grammaticalization (2 vol.), Amsterdam-Philadelphia, John Benjamins, pp. 17-35.

KALtenbÖCK, G. (2007), "Position, prosody, and scope: the case of English comment clauses ", Vienna English Working Papers, n $16 / 1$, pp. 3-38.

Koch, P. \& Österreicher, W. (2001), «Gesprochene Sprache und geschriebene Sprache. Langage parlé et langage écrit », in Holtus, G., Metzeltin, M. \& Schmitt, C. (eds), Lexikon der romanistischen Linguistik, n¹-2, pp. 584-627.

LAmbrecht, K. (1994), Information Structure and Sentence Form. Topic, Focus and the Mental Representations of Discourse Referents, Cambridge, Cambridge University Press.

LAmbrecht, K. (2000), «Prédication seconde et structure informationnelle. La relative de perception comme construction présentative », Langue Française, n²127, pp. 49-66.

LANGACKER, R. W. (1987), Foundations of cognitive grammar, Stanford, Stanford University Press.

LEHMAnN, C. (1995), Thoughts on Grammaticalization, Munich, LINCOM-Europa.

LEWIS, D. (2006), «Discourse particle : a discourse-pragmatic category », in Fischer, K. (ed.), Approaches to Discourse Particles, Oxford, Elsevier, pp. 43-60. 
LEWIS, D. 2011), «A discourse-constructional approach to the emergence of discourse connectives in English », Linguistics, n²9/2, pp. 415-443.

LindSTRÖM, J. (2001), "Inner and outer syntax of constructions: The case of the $x$ och $x$ construction in Swedish» (Paper presented in the panel on Pragmatic aspects of frame semantics and construction grammar, 7th International Pragmatics Conference, Budapest July 9-14 2000.). Preliminary version published at project site Grammar in Conversation : a Study of Swedish.

LINDSTRÖM, J. \& KARLSSON, S. (2005), «Verb-first constructions as a syntactic and functional resource in (spoken) Swedish », in Nordic Journal of Linguistics, 28/1, pp. 1-35.

MARANDin, J.-M. (1999), Grammaire de lincidence (Manuscrit non publié). http://www.llf.cnrs.fr/fr/Marandin/]

Marchello-NiziA, C. (2006), Grammaticalisation et changement linguistique, Bruxelles, De Boeck Université - Duculot, Coll. Champs linguistiques.

Nespoulous, J.-L. (2010), «Référence et modalisation : de la dissociation de leur gestion psycholinguistique et neuropsycholinguistique dans le discours aphasique », in Neveu, F., Durand, J., Klingler, T., Prévost, S. \& Muni-Toké, V. (éds), Actes du CMLF 2010 (2e Congrès Mondial de Linguistique Française, 12-15 juillet 2010, New Orleans, Etats-Unis).

Norde, M. (2009), Degrammaticalization, Oxford, Oxford University Press.

Perrin, L. (2008), «Le sens montré n’est pas dit », in Birkelund, M., Hansen, M.-B. \& Norén, C. (dir.), L'énonciation dans tous ses états. Mélanges offerts à Henning Nolke à l'occasion de ses soixante ans, Bern, Peter Lang, pp. 157-187.

PrÉVOST, S. (2001), La postposition du sujet en français aux $15^{e}$ et $16^{e}$ siècles : une approche sémanticopragmatique, Paris, Editions du CNRS.

PRÉvost, S. (2003a), « Détachement et topicalisation : des niveaux d'analyse différents », Cabiers de Praxématique, $\mathrm{n}^{\circ} 40$, pp. 97-126.

PrÉvost, S. (2003b), « La grammaticalisation : unidirectionnalité et statut », Le Français Moderne, $\mathrm{n}^{\circ} 71 / 2$, p. 144-166.

SCHOuRup, L. (1999), « Discourse markers », Lingua, n¹07, pp. 227-265.

SCHNEIDER, S. (2007), Reduced parenthetical clauses as mitigators : a corpus study in Spoken French, Italian and Spanish. Amsterdam-Philadelphia, John Benjamins.

Thompson, S.A. \& MulaC, A. (1991), «A quantitative perspective on the grammaticization of epistemic parentheticals in English», in Heine, B. \& Traugott, E.C. (eds), Approaches to Grammaticalization, Volume II, Amsterdam-Philadelphia, John Benjamins, pp. 313-329.

Torres Cacoullos, R. \& Walker, J. A. (2009), « On the persistence of grammar in discourse formulas : A variationist study of that $»$, Linguistics, $\mathrm{n}^{\circ} 47 / 1$, pp. 1-43.

Torres Cacoullos, R. \& Walker, J. A. (2011), «Collocations in grammaticalization and variation », in Heine, B. \& Narrog, H. (eds), The Oxford Handbook of Grammaticalization (Chapter 18), Oxford, Oxford University Press. 
Catherine Bolly

TraugotT, E.C. (1982), «From propositional to textual and expressive meanings : Some semantic-pragmatic aspects of grammaticalization ", in Lehmann, W.P. \& Malkiel, Y. (eds), Perspectives on historical linguistics, Amsterdam, John Benjamins, pp. 245-271.

TRAugOTT, E.C. (1997), «The role of the development of discourse markers in a theory of grammaticalization » (Communication à ICHL XII, Manchester 1995, version du 11/97).

TRAUGOTT, E.C. (2001), «Legitimate counterexamples to unidirectionality » (Article présenté à l’Université de Freiburg, le 17 octobre 2001).

TRAugotT, E.C. (2008), «'All that he endeavoured to prove was ...': On the emergence of grammatical constructions in dialogual and dialogic contexts", in Cooper, R. \& Kempson, R. (eds), Language in Flux : Dialogue Coordination, Language Variation, Change and Evolution, London, Kings College Publications, pp. 143-177.

TRAugOtT, E.C. (2010), " (Inter)subjectivity and (inter)subjectification : A reassessment », in Davidse, K., Cuyckens, H. \& Vandelanotte, L. (eds), Subjectification, Intersubjectification and Grammaticalization, Berlin-New York, Mouton de Gruyter, pp. 29-74.

Urmson, J. (1952), «Parenthetical verbs », Mind, nº1, pp. 480-496.

VAN BOGAERT, J., (2011), «I think and other complement-taking mental predicates : A case of and for constructional grammaticalization », Linguistics, $n^{\circ} 49 / 2$, pp. 295-332.

Willems, D. \& Blanche-Benveniste, C. (2010), «Verbes “faibles” et verbes à valeur épistémique en français parlé : il me semble, il paraît, j'ai l'impression, on dirait, je dirais », in Iliescu, M., Siller-Runggaldier, H. \& Danler, P. (dir.), Actes du XXVe CILPR Congrès International de Linguistique et de Philologie Romanes, Innsbruck, 3- 8 septembre 2007, BerlinNew York, De Gruyter, pp. 565-576. 